\title{
Three-dimensional detection of sequence of strokes using confocal microscope
}

\author{
Manisha Mann ${ }^{1}$, Seema Rani Pathak ${ }^{1^{*}}$ and Sudhir Kumar Shukla ${ }^{2}$
}

\begin{abstract}
Background: In the present study, three-dimensional application of confocal microscope is explored. The aim is to determine the sequence of intersecting lines written on a questioned document as a non-destructive technique. The authors have tested the Nikon confocal A1 microscope on two major criteria. The criterion 1 focuses on the analysis of homogenous intersecting lines, whereas the criterion 2 focuses on the effect of pen pressure on the sequence of the strokes. Fifty-four and 42 samples from criterion 1 and criterion 2 respectively were examined. All the intersecting strokes were homogeneous and in either black- or blue-coloured ink. They were made from glycolbased (ballpoint pen) and liquid-based (fountain pen and gel pen) inks.

Result: The authors were able to determine the correct sequence of homogeneous intersecting lines in 81 and $83 \%$ of the average data of samples for criterion 1 (the analysis of homogenous intersecting lines) and criterion 2 (the effect of pen pressure on the sequence of the strokes) respectively.

Conclusion: The confocal microscope proved to be a reliable and precise technique for analysing the homogeneous intersected lines made by any pen pressure beyond all the errors during interpretation.
\end{abstract}

Keywords: Sequence of intersecting lines, Homogenous ink, Pen pressure, Glycol-based ink, Liquid-based ink

\section{Background}

Determining the correct chronological order of sequence of strokes and finding the appropriate technique for examination of such cases is a challenging task. During the investigation of fraudulent documents, the sequence of two ink strokes can impart vital information (Berx and Kinder 2002). Many conventional techniques present in the domain of Forensic Questioned Document, namely 2-D imaging (Saini et al. 2009; Fabianska and Kunicki 2003) and lifting technique (Leung and Leung 1997; Koons 1985; Igoe and Reynolds 1982) can be used to determine the sequence of strokes. 2D imaging technique misses out on the accuracy aspect when the intersecting pen strokes are made from homogeneous strokes (Spagnolo 2006). The main disadvantage of $2 \mathrm{D}$ imaging technique is that the darker or heavier ink line may appear above the lighter ink line, irrespective of the correct order of the stroke. It may create an

\footnotetext{
* Correspondence: seemarpathak@gmail.com

${ }^{1}$ Department of Chemistry, Bio-chemistry and Forensic Sciences, Amity

School of Applied Sciences, Amity University Haryana Gurugram, Haryana

122413, India

Full list of author information is available at the end of the article
}

optical illusion in the mind of the examiner leading to errors in the interpretation (Bojko et al. 2008). Further, the dispersion of ink particles from the previous ink line to the latter ink line creates doubts in the mind of the examiner (Claybourn and Ansell 2000).

To deal with homogeneous intersecting strokes, non-contact techniques, also known as 3D imaging techniques are used. These techniques are a reliable alternative to conventional 2D techniques and are becoming popular in the domain of Forensic Questioned Document (Heikkinen et al. 2010). A few studies have used laser scanning confocal microscopy and 3D laser profilometry to determine the sequence of strokes (Berx and Kinder 2002; Heikkinen et al. 2010; Cheng et al. 1998). One of the most significant advantages of 3D imaging technology is that it allows the examiner to analyse the questioned document further under other techniques without any damage or modification to documents (Spagnolo et al. 2005). Additionally, 3D imaging techniques do not require any sample preparation. These are non-destructive in nature and are not affected by the type of pen, type and colour of ink, and pen pressure applied while making a fraudulent document (Spagnolo 
2006; Ahvenainen et al. 2010; Poulin 1996). Study inferences the differences in the school of thoughts on the effect of pen pressure on 3D imaging techniques (Berx and Kinder 2005; Montani et al. 2012). The pen pressure can profoundly impact the underlying paper surface. Amount of pressure applied to the tip of the pen during the process of writing creates grooves in the paper surface. The higher the pen pressure, the deeper are the grooves, and in the case of low pen pressure, shallow grooves are formed (Ahvenainen et al. 2010).

In the current study, the authors aim to evaluate the efficiency of 3D imaging. The power of Nikon A1 confocal microscope to overcome the investigation challenges of homogeneity of crossed strokes and the strokes from low pen pressure is examined. To obtain conclusive results related to factors as mentioned above, the authors have studied two objectives-examining the sequence of homogenous intersecting strokes (criterion 1) and determine the effect of pen pressure on the sequence of strokes (criterion 2).

\section{Availability of data and material}

All data generated and analysed during this study has been included in this published article.

\section{Material and methods}

\section{Instrument}

Nikon A1 confocal microscope was used to conduct the experiments. It comprises of a resonant scanner which together with a non-resonant scanner provides high resolution for capturing images. It uses four PMT (photomultiplier tube) detectors which allow excitation of the sample up to four lasers, which means that simultaneous excitation of the sample can be done by using up to four lasers at a time (Heikkinen et al. 2010). All the samples were examined at 415-495 nm wavelength. The step size to analyse each sample was $10 \mu \mathrm{m}$, with 51 frames captured for each step size producing a three-dimensional image ( $z$ sectioning). Two types of images were obtained for each analysed sample-the first one was three-dimensional and the second was an image captured in all the channels like TD (transmission detector), DAPI ('6-diamidino-2-phenylindole) and FITC (fluorescein isothiocyanate). A picture of a Nikon A1 confocal microscope is depicted in Fig. 1.

\section{Samples}

\section{Sample preparation for criterion 1}

Following parameters were used in making the samples
a. Number of samples examined: 54
b. Paper used: A4 sheet Trident Spectra Photocopier Papers
c. Colour of ink: black and blue. (9 samples each)

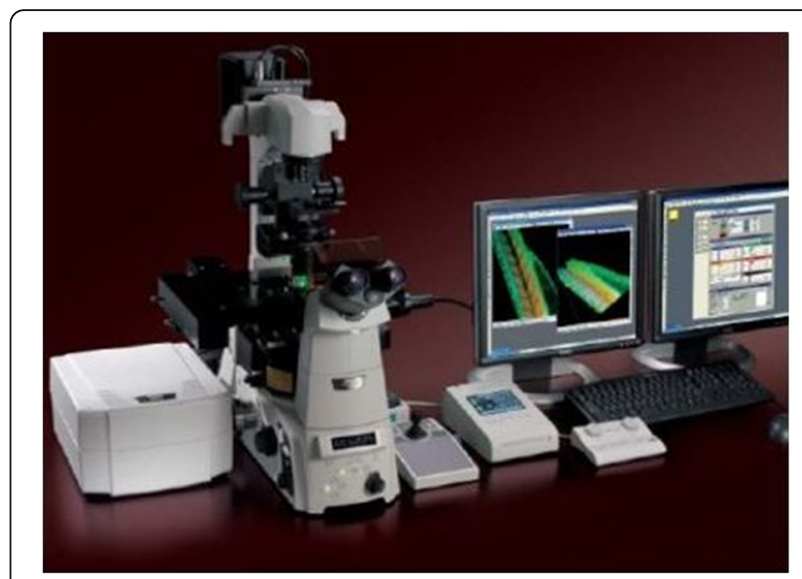

Fig. 1 Nikon A1 confocal microscope

d. Nature of ink: glycol-based (ballpoint pen) and liquid-based (fountain pen and gel pen)

e. Brand of writing media: ballpoint pen-Cello Pinpoint, gel pen-Cello Butterflow and fountain pen: Chelpark fountain pen. (18 samples each)

f. Dimensions of the sample paper: $7 \times 3 \mathrm{~cm}$.

g. Time between intersection: immediate (within $5 \mathrm{~s}$ )

Figure 2 explains the protocol followed in making the samples for criterion 1.

\section{Sample preparation for criterion 2}

Following parameters were used in making the samples

a. Number of samples examined: 42

b. Paper used: A4 sheet Trident Spectra Photocopier Papers

c. Pen pressure: light, medium and heavy (7 samples each)

d. Colour of ink used: black

e. Nature of ink: glycol-based (ballpoint pen) and water-based (gel pen)

f. Brand of writing media: ballpoint pen-Cello Pinpoint, gel pen-Cello Butterflow (21 samples each)

g. Dimensions of the sample paper: $7 \times 3 \mathrm{~cm}$

h. Time between intersection: immediate (within $5 \mathrm{~s}$ )

Figure 3 explains the protocol followed in making samples for criterion 2 .

\section{Sample details}

All samples for criterion 1 and criterion 2 were prepared from the same model (Fig. 4a). In each sample, firstly 5000 was written, and the signature was placed above 5000. Then, one was added between 5 and the first 0 ; it means the numbers 5 and 0 are below the stroke of the 


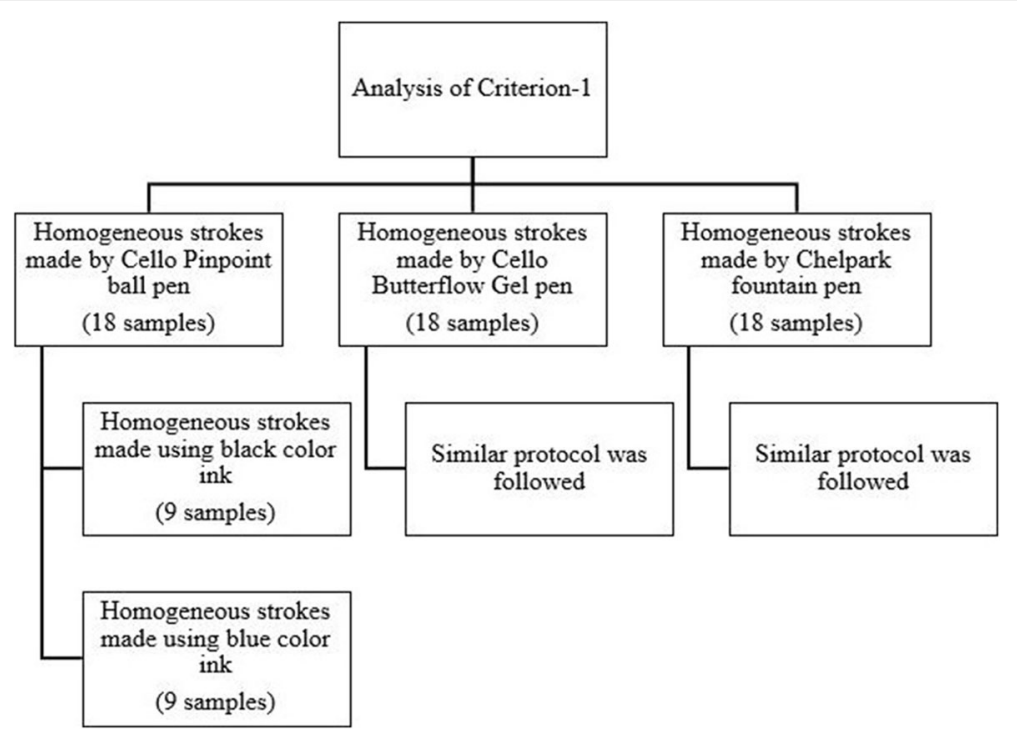

Fig. 2 Graphical representation illustrating the protocol of producing samples for criterion 1

signature, and 1 is above the stroke of the signature. Figure $4 \mathrm{~b}$ represents the sequence in which the area of interest was created. In the figure, the sequence is represented by 1, 2, 3 and 4 . The prepared samples were then placed on top of standard glass slides, and using transparent cello tape, the ends of the sample were stuck to a glass slide. Fixing of the sample on the glass slide was done to ensure that the sample does not get displaced and focused images can be captured under the Nikon A1 confocal microscope.

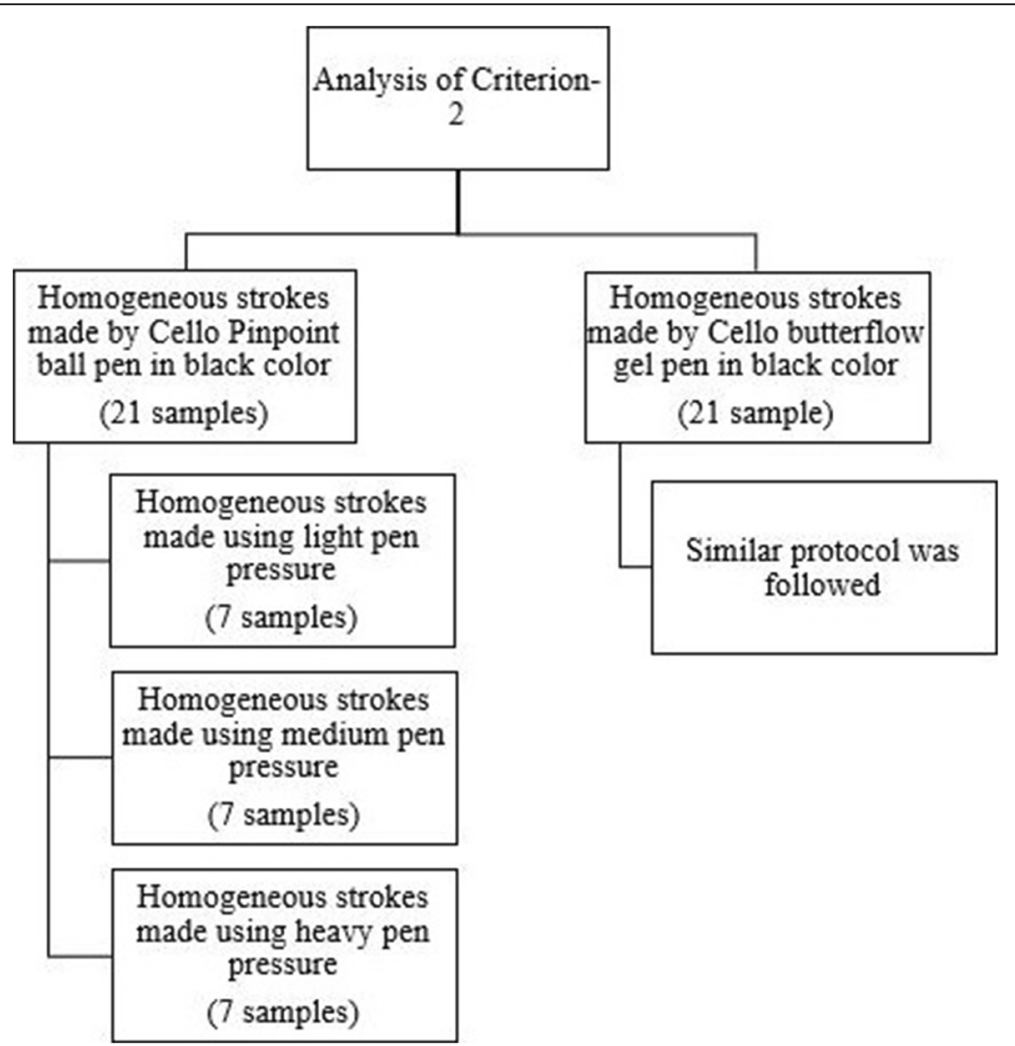

Fig. 3 Graphical representation illustrating the protocol of producing samples for criterion 2 

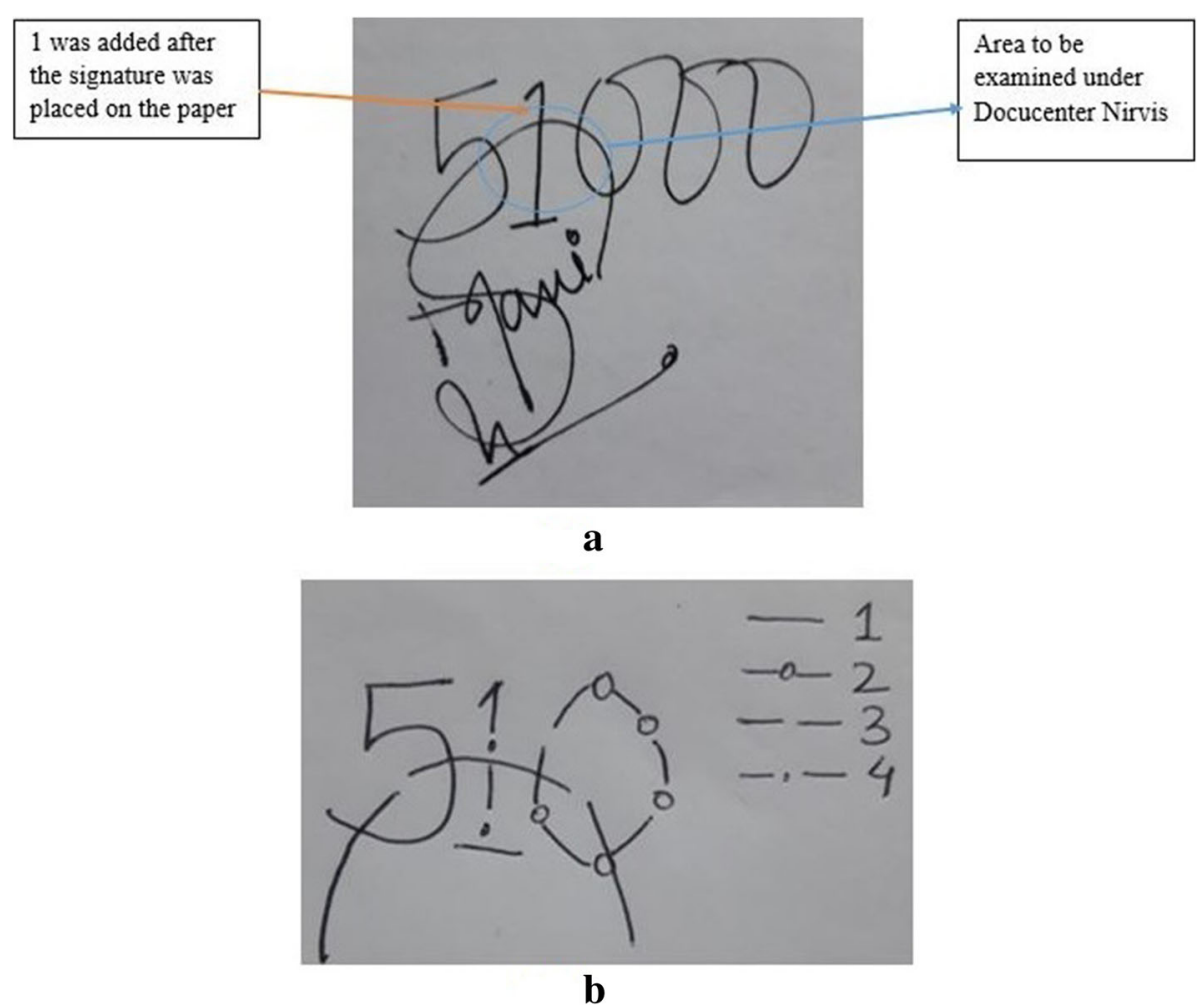

Fig. 4 a Image of the model sample. $\mathbf{b}$ Image of the sequence

\section{Results and discussion}

\section{Analysis of the intersecting homogenous strokes}

After examining the samples with a Nikon A1 confocal microscope, it is evident that the Nikon A1 confocal microscope can accurately determine the sequence of the intersecting lines made from the same type and colour of ink. The results were labelled as confident and not confident. These terms indicate positive to negative results. About $80 \%$ of the samples gave confident results, and the rest belonged to the non-confident category. The results of criterion 1 are presented in Table 1.

Examination of the sequence of homogenous strokes is one of the most concerned areas in forensic document examination. Because of the degree of human subjectivity involved, it has the potential to create doubt in the mind of the researcher and may lead to an incorrect conclusion. The three-dimensional analysis of intersecting strokes assisted the study by helping in forming a definite opinion on the determination of the sequence of the strokes made by homogenous inks. Two types of images were captured using the Nikon A1 confocal microscope: the first one is a three-dimensional image (Figs. 5b, 6b and 7b), and the second one was taken in different channels like DAPI, FITC and TD. The best results were seen in the TD channel (Figs. 5a, 6a and 7a). Three types of writing instrument were used for the homogenous intersected strokes-ballpoint pen, the gel pen and the fountain pen. Black and blue colours were used. An optimum amount of pen pressure was applied for the samples of criterion 1 . The three-dimensional image was very valuable, as the grooves made by the writing instrument on the paper were visible and the sequence of pen strokes made on the paper could be identified. The sequence of the intersecting lines was determined by examining the continuity of the latter ink line over the former ink line.

Table 1 Results of criterion 1 observed under Nikon A1 confocal microscope

\begin{tabular}{lllll}
\hline S. no. & Type of intersection & Confident result & Non-confident result & Success rate (\%) \\
\hline 1. & BA-BP & 9 & 0 & 100 \\
2. & BA-FP & 7 & 2 & 77.7 \\
3. & BA-GP & 9 & 0 & 100 \\
4. & BU-BP & 9 & 0 & 100 \\
5. & BU-FP & 6 & 3 & 66.6 \\
6. & BU-GP & 3 & 6 & 33.3 \\
\hline
\end{tabular}




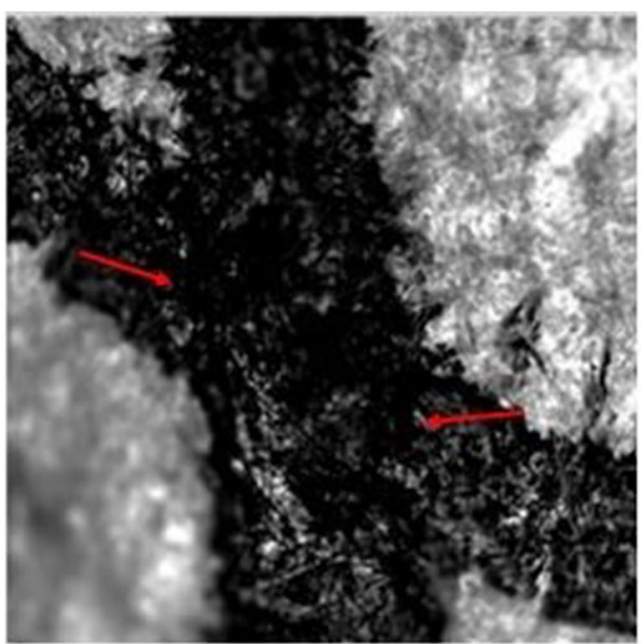

a

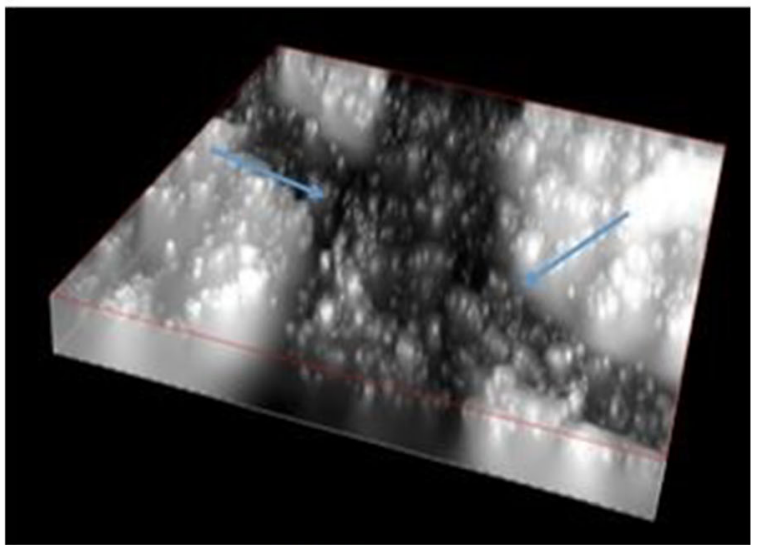

b

Fig. 5 Black Cello Pinpoint pen intersecting strokes. a 2D figure. b 3D figure

Presence of edge marks in the latter pen line was observed which indicated that it interrupted the former ink line (Berx and Kinder 2002).

In the Figs. 5a, 6a and 7a, the interpretation was made on the basis of continuity of the second ink line over the first ink line at the point of intersection. The continuity phenomena of the latter ink line are marked with the red-coloured arrow. In the 3D images, former ink line can be easily seen beneath the latter ink line, and the difference in the levels of both the ink line at the point of intersection is marked with the blue-coloured arrow in the Figs. 5b, $6 \mathrm{~b}$ and $7 \mathrm{~b}$.

Since the time of intersection between the two lines is less than $5 \mathrm{~s}$, the ink particles of the former ink line get dragged into the latter ink line. This could have led to the non-confident results. In this case, the dragged ink particles were of the same type and colour, thus making the examination even more difficult. Hence, it was not possible to differentiate the edge marks of both the ink lines. The success

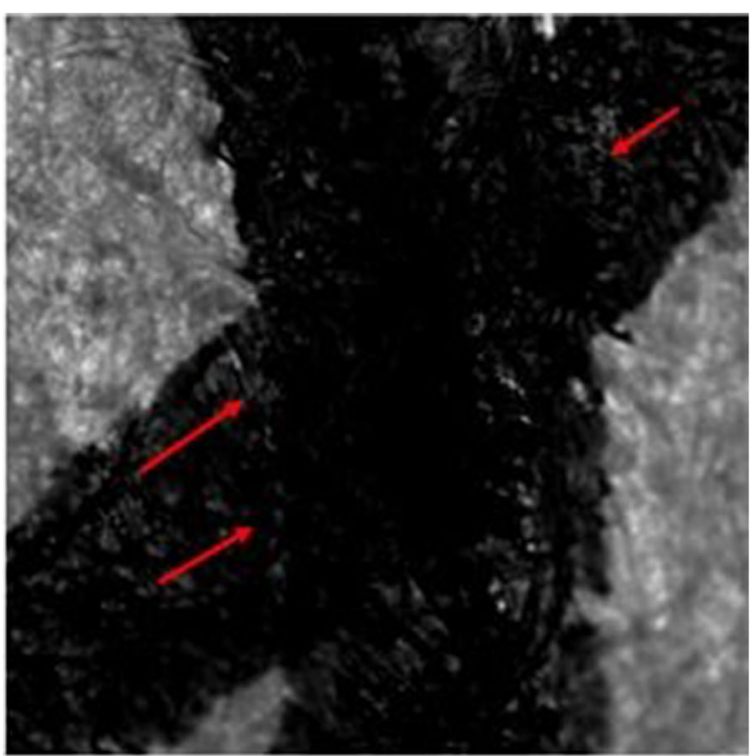

a

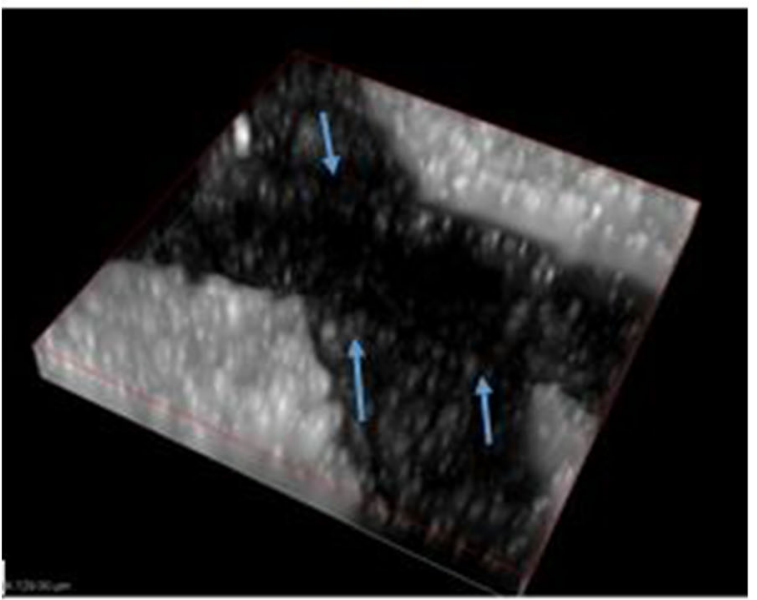

b

Fig. 6 Black Cello Butterflow pen intersecting strokes. a 2D figure. b 3D figure

rate of the positive examination of the sequence of strokes is in the following order-ballpoint pen $>$ fountain pen $>$ gel pen. It was observed that the intersections made by black-coloured ink gave better results as compared to the intersections made by blue-coloured ink.

During the examination of the samples made from blue gel pen, it was observed that the colour of the ink appeared as off-white on the surface of the paper, so differentiation between the ink line of blue gel pen and paper could not be adequately made. Figure $8 \mathrm{a}, \mathrm{b}$ in TD channel and $3 \mathrm{D}$ image respectively depict a blue gel pen sample observed under Nikon A1 confocal microscope. In both the images, one ink line is marked with a green arrow and 


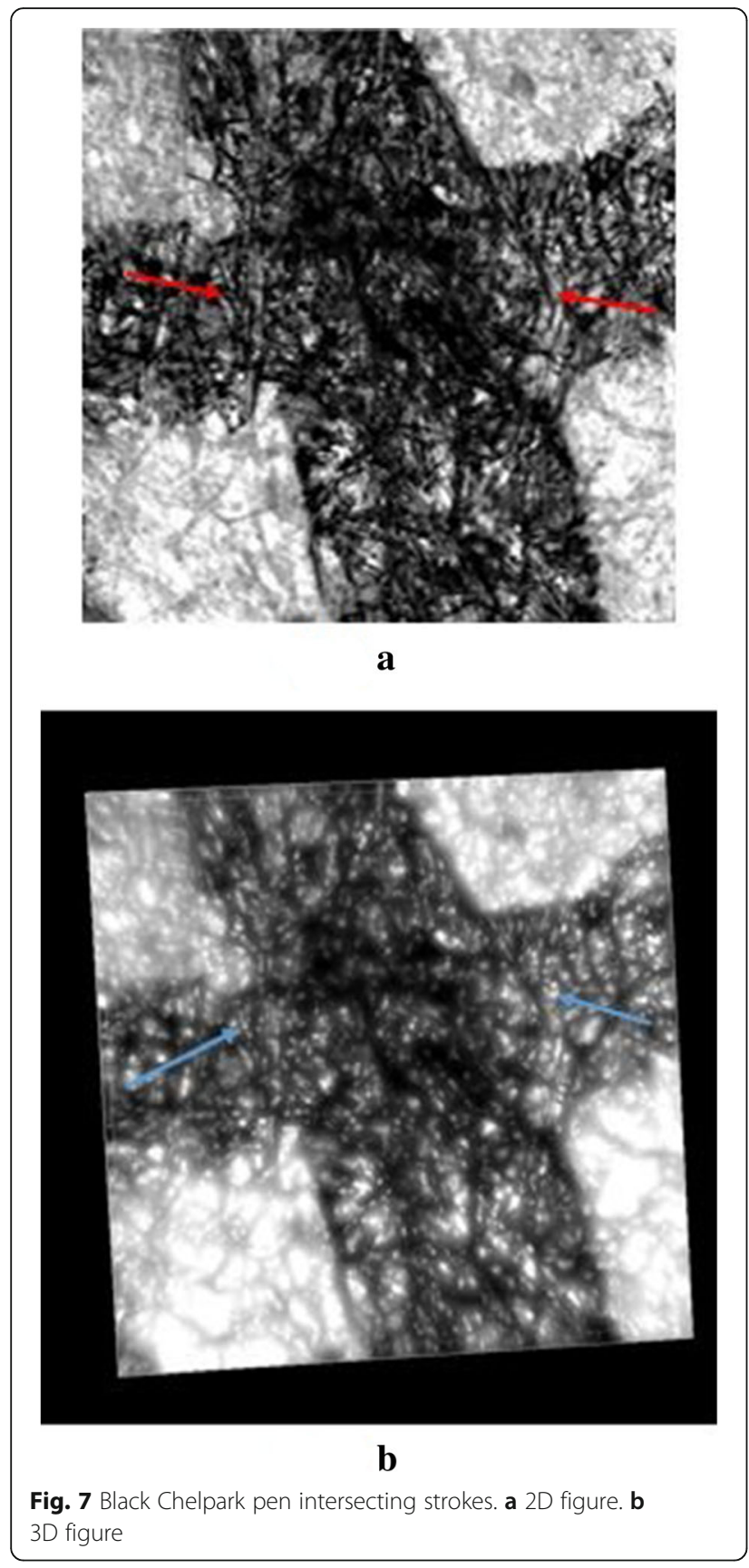

the other one with a black arrow. Therefore, the results were termed as the non-confident.

Confocal microscope enabled the authors to take high-resolution three-dimensional images of small sections of the samples.

\section{Effect of pen pressure on sequence of the strokes}

The second aspect which was analysed was the effect of different types of pen pressure on the sequence of strokes. The impact of the pen pressure on the sequence of the strokes can be seen in Table 2. Total 42 samples

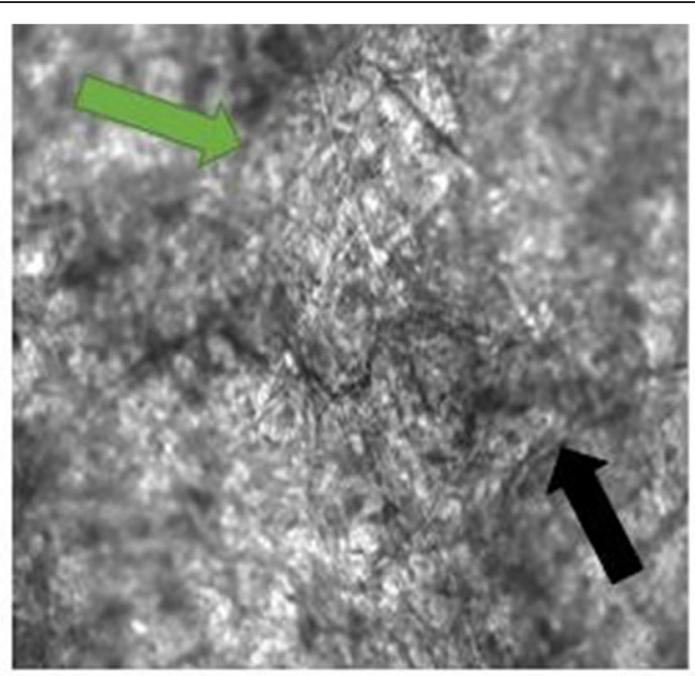

a

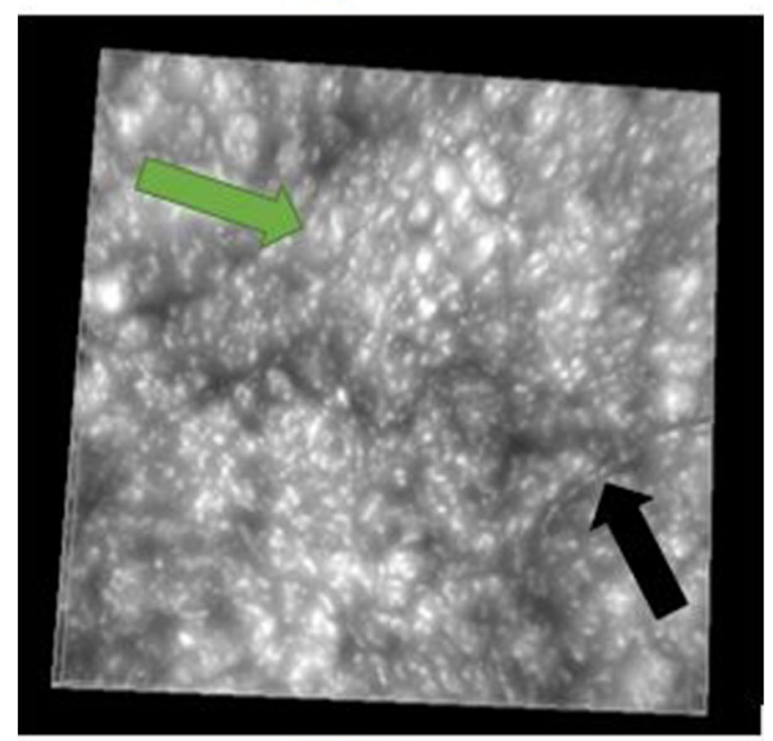

b

Fig. 8 Blue Cello Butterflow pen intersecting strokes. a 2D figure. b 3D figure

made from black-coloured ink were examined under the Nikon A1 confocal microscope. Samples made with heavy pen pressure gave the best results followed by samples made with medium and light pen pressure. The authors were able to find the correct chronological sequence of homogeneous intersected lines in $76 \%$ of the samples made from black Cello Pinpoint ball pen and $90 \%$ of the samples made from black Cello Butter flow gel pen. The rest of the samples gave the inconclusive result. Two types of images were captured using the Nikon A1 confocal microscope; the first one is a three-dimensional image (Figs. 9b, 10b and 11b), and the second one was taken in different channels like DAPI, 
Table 2 Results of criterion 2 observed under Nikon A1 confocal microscope

\begin{tabular}{lllll}
\hline Type of pressure & Type of intersection & Confident result & Non-confident result & Success rate (\%) \\
\hline Light pen pressure & BA-BP & 5 & 2 & 1 \\
Medium pen pressure & BA-GP & 6 & 2 & 1.4 \\
BA-BP & BA-GP & 5 & 1 & 75.7 \\
Heavy pen pressure & BA-BP & 6 & 1 & 85.7 \\
& BA-GP & 6 & 0 & 85.7 \\
\hline
\end{tabular}

FITC and TD. The best results were seen in the TD channel (Figs. 9a, 10a and 11a).

Every writer applies a specific amount of pressure while writing, which is characteristic of an individual's handwriting. Pen pressure can be classified into three categories-light, medium and heavy. These pen pressure were applied while making the intersecting lines in

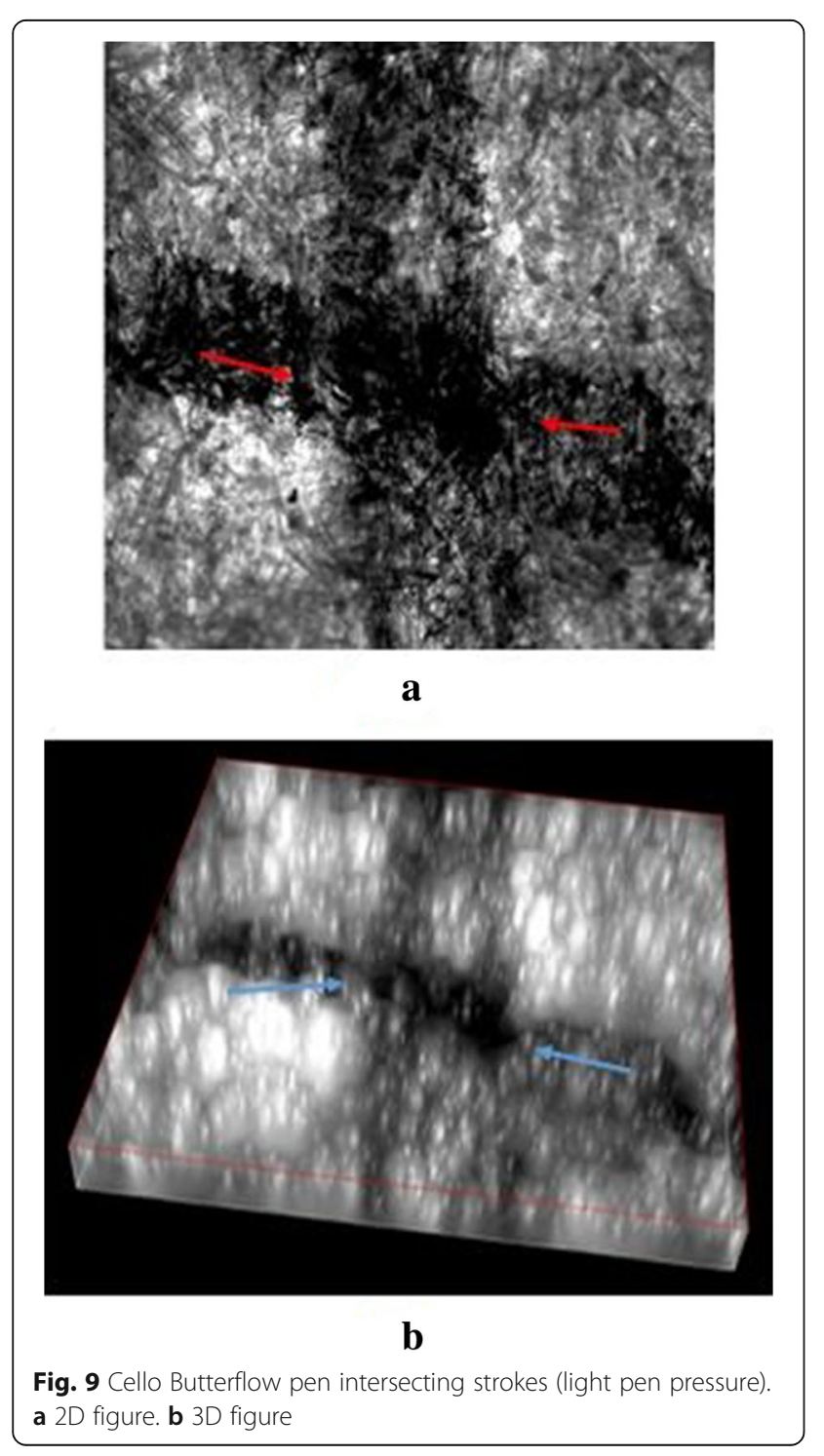

a sample. The pressure of the pen while writing is related to the speed of writing. Usually, in the case of light pen pressure, the speed will be relatively fast and will decrease with increase in the amount of pen pressure. Heavy pen pressure had the maximum effect on the sequence of strokes. In this case, the writing will be slow, and the amount of ink entering into the paper will be

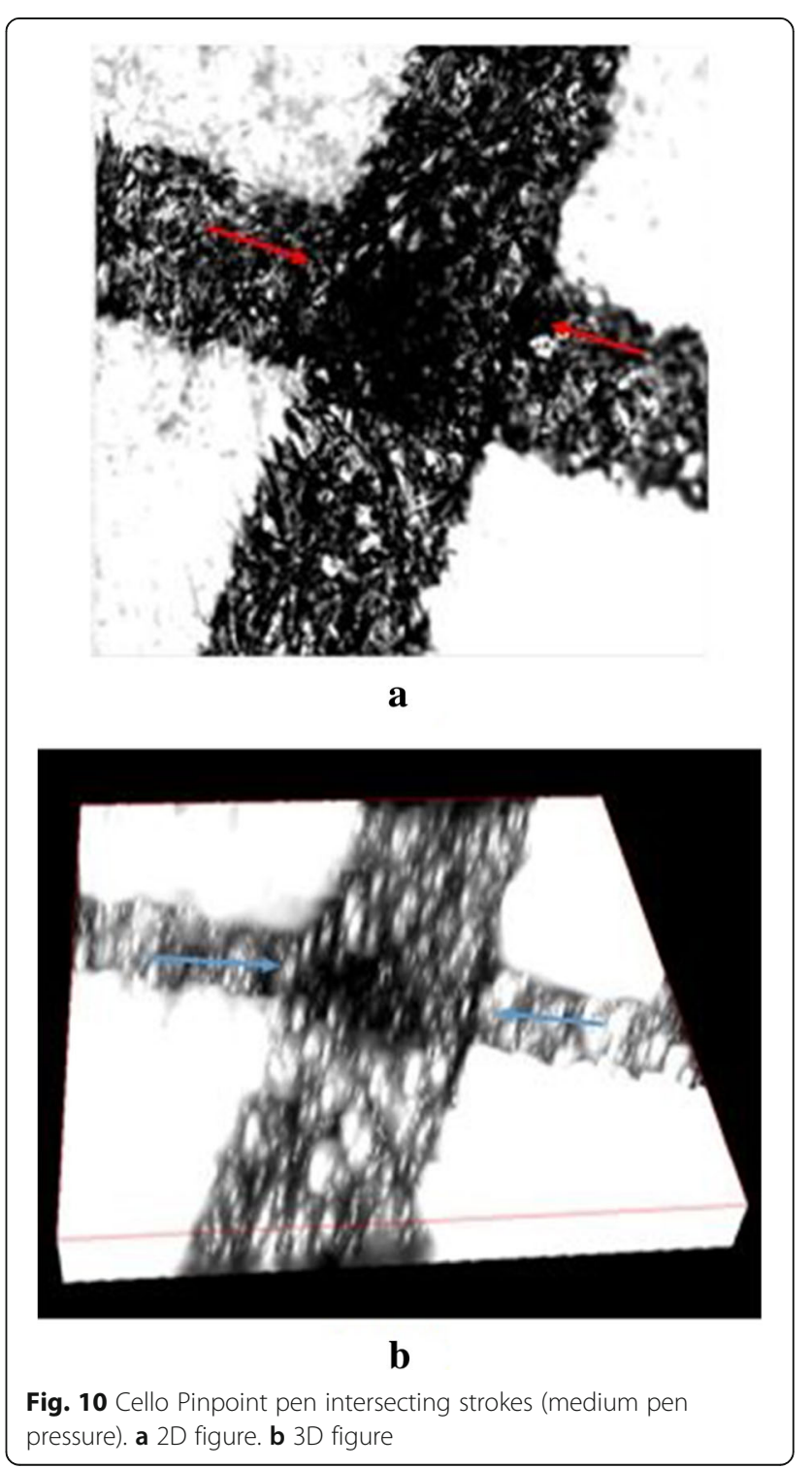




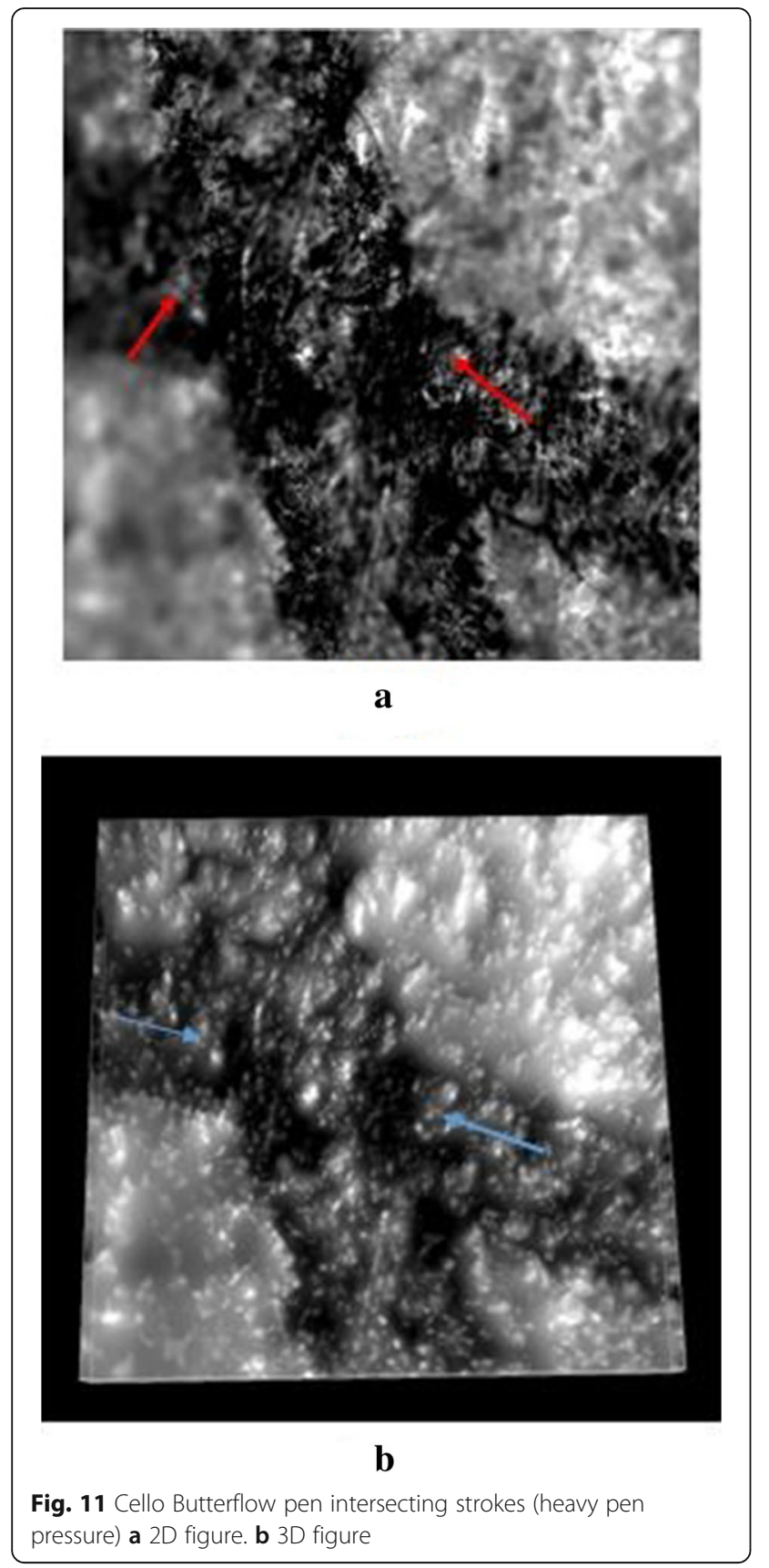

more as compared to fast writing. Generally, the heavy deposition of the ink makes the examination process even more difficult as under the normal microscope the heavier ink line is seen on the top, even if the ink line is not above the former ink line (Ellen 2006). It was observed that the said ink deposition factor did not affect the examination process and the authors were able to find the exact order of intersected lines using a Nikon A1 confocal microscope. In the Figs. 9a, 10a and 11a, the interpretation was made on the basis of continuity of the second ink line over the first ink line at the point of intersection. The continuity phenomena of the latter ink line are marked with a red-coloured arrow. In the 3D figures, former ink line can be easily seen beneath the latter ink line, and the difference in the levels of both the ink line at the point of intersection is marked with the blue-coloured arrow in the Figs. 9b, 10b and 11b. It was also observed that the effect of medium pen pressure and light pen pressure on the examination of the sequence of the strokes was minimal, despite the fact that dragging of pigment from one line to another line was observed. The three-dimensional images were found to be very useful in determining the correct chronological sequence of the intersecting lines irrespective of the amount of applied pen pressure while writing.

Pen pressure formed a groove on the surface of the paper, and the depth of the groove depends on the amount of pen pressure applied. The higher the pen pressure, the deeper will be the depth of the groove and higher are the chances of determining the sequence of strokes (Daéid et al. 2008). The writing instrument used in making the samples also affected the groove formed from the pen pressure. It was observed that the grooves formed by ballpoint pen were wider and deeper due to the size of the ball sphere present on its tip, as compared to the gel pen.

\section{Conclusion}

Overall, the Nikon A1 confocal microscope is a versatile instrument for the analysis of the sequence of strokes. The properties of the confocal microscope assisted in the examination process, especially the three-dimensional images were helpful in examining the sequence of the strokes made by homogeneous ink. The three-dimensional analysis also proved advantageous in determining the exact sequence of intersecting strokes irrespective of the amount of pen pressure applied while writing. The three-dimensional images aided in visualising the two-dimensional features of intersecting lines using three-dimensional features like depth of grooves formed by the tip of the pen. The confocal microscope is highly recommended for examining the sequence of the strokes made in black-coloured ink. The confocal microscopy is a total non-destructive technique and requires very minimal sample preparation. As discussed in the previous studies, it gives verifiable results even in low pen pressure. The only disadvantage of the confocal microscope is that it takes time to analyse the sample. Further studies are being conducted to analyse the intersecting strokes made using different brands of blue gel pens.

\section{Abbreviations}

BA: Black colour; BP: Ballpoint pen; BU: Blue colour; DAPI: '6-diamidino-2phenylindole; FITC: Fluorescein isothiocyanate; FP: Fountain pen; GP: Gel pen; TD: Transmission detector

Acknowledgements

Not applicable. 


\section{Funding}

There was no funding authority as of, the study is part of an ongoing Ph.D. research.

\section{Availability of data and materials}

All data generated or analysed during this study are included in this published article.

\section{Authors' contributions}

SRP and SKS assisted in the research article. All the experimental work is conducted by MM. All authors read and approved the final manuscript.

\section{Ethics approval and consent to participate}

No ethical approval and consent is required as author prepared the samples. No ethical approval and consent is required as there was no involvement of human participants, human data or human tissue.

\section{Consent for publication}

The manuscript does not contain any person's data in any form. On behalf of all the authors, I consent that the said manuscript submitted can be published in your esteemed journal.

\section{Competing interests}

The authors declare that they have no competing interests.

\section{Publisher's Note}

Springer Nature remains neutral with regard to jurisdictional claims in published maps and institutional affiliations.

\section{Author details}

${ }^{1}$ Department of Chemistry, Bio-chemistry and Forensic Sciences, Amity School of Applied Sciences, Amity University Haryana Gurugram, Haryana 122413, India. ${ }^{2}$ Amity Institute of Forensic Sciences (AIFS), Amity University, Noida, Uttar Pradesh 201301, India.

Received: 11 August 2018 Accepted: 11 March 2019

Published online: 20 March 2019

\section{References}

Ahvenainen P, Kassamakov I, Hanhijärvi K et al (2010) Csi Helsinki: Swli in forensic science: comparing tool marks of diagonal cutting pliers. Rev Quant NonDestr Eval 29:2084-2091

Berx V, Kinder JD (2002) A 3D view on the 'crossing lines' problem in document examination. In: Proc. SPIE 4709, pp 102-110

Berx V, Kinder JD (2005) The application of profilometry in the analysis of the line crossings. J Am Soc Quest Doc Exam 8:1-8

Bojko K, Roux C, Reedy BJ (2008) An examination of the sequence of intersecting lines using attenuated total reflectance Fourier transform infrared spectral imaging. J Forensic Sci 53:1458-1467

Cheng KC, Chao CH, Jeng BS, Lee ST (1998) A new method of identifying writing sequence with the laser scanning confocal microscope. J Forensic Sci 43: 348-352

Claybourn M, Ansell M (2000) Using Raman spectroscopy to solve crime: inks, questioned documents and fraud. Sci Justice 40:261-271

Daéid NN, Whitehead L, Allen M (2008) Examining the effects of paper type, pen type, writing pressure and angle of intersection on white and dark dominance in ESDA impressions of sequenced strokes - an application of the likelihood ratio. Forensic Sci Int 181:32-35

Ellen D (2006) Scientific examination of documents: methods and techniques, 3rd edn. Taylor and Francis Group, USA

Fabianska E, Kunicki M (2003) Raman spectroscopy as a new technique for determining the Asequence of intersecting lines. Probl Forensic Sci 53:60-73

Heikkinen V, Barbeau C, Kassamakov I et al (2010) Determining the chronological order of crossing lines using 3D imaging techniques. In: Optics and Photonics for Counterterrorism and Crime Fighting VI and Optical Materials in Defence Systems Technology VII

Igoe TJ, Reynolds BL (1982) A lifting process for determining the writing sequence of two intersecting ball-point pen strokes. Forensic Sci Int 20:201205

Koons RD (1985) Sequencing of intersecting lines by combined lifting process and scanning electron microscopy. Forensic Sci Int 27:261-276
Leung SC, Leung YM (1997) A systematic study of the lifting technique for determining the writing sequence of intersecting ball pen strokes. Sci Justice 37:197-206

Montani I, Mazzella W, Guichard M, Marquis R (2012) Examination of heterogeneous crossing sequences between toner and rollerball pen strokes by digital microscopy and 3-D laser Profilometry. J Forensic Sci 57:997-1002

Poulin G (1996) Establishing the sequence of strokes: the state of the art. Int J Forensic Doc Examiners 2:16-32

Saini K, Kaur R, Sood N (2009) A study for establishing the sequence of superimposed lines: inkjet versus writing instruments. Forensic Sci Int 193:14-20

Spagnolo GS (2006) Potentiality of 3D laser profilometry to determine the sequence of homogenous crossing lines on questioned documents. Forensic Sci Int 164:102-109

Spagnolo GS, Simonetti C, Cozzella L (2005) Determination of the sequence of line crossings by means of 3D laser profilometry. In: Optical Security Systems, Proc of SPIE

\section{Submit your manuscript to a SpringerOpen ${ }^{\circ}$ journal and benefit from:}

- Convenient online submission

- Rigorous peer review

- Open access: articles freely available online

- High visibility within the field

- Retaining the copyright to your article

Submit your next manuscript at $>$ springeropen.com 